\title{
PHEIDOLE NASUTOIDES, A NEW SPECIES OF COSTA RICAN ANT THAT APPARENTLY MIMICS TERMITES
}

\author{
By BERT HÖLLdobleR ${ }^{1}$ AND EDWARd O. WILSON ${ }^{2}$
}

Mimicry is a commonplace among species of ants (Hölldobler and Wilson, 1990). However, to our knowledge no ant or any other social hymenopteran has been recorded that mimics termites, despite the fact that many termite species have formidable defenses against both vertebrates and invertebrates that would seemingly make them ideal models. In 1985, at the La Selva Field Station and Biological Reserve of the Organization for Tropical Studies, in northeastern Costa Rica, we discovered a new species of Pheidole whose major workers in life astonishingly resembled those of Nasutitermes. These strange-looking ants were nesting in the low arboreal zone at the edge of secondary rain forest, in a habitat especially favored by Nasutitermes. Our critical study was limited to a brief observation of the Pheidole at the nest site and in the laboratory. On three later visits to this locality during the subsequent five years we searched the vicinity of the original find and widely elsewhere through the La Selva station, in an attempt to locate additional colonies and conduct more detailed studies. We were never successful; the new Pheidole remains known only from the original colony. Because of its unique qualities and possible unusual biological significance, we have chosen to describe it now, and with the description the hypothesis that this ant is indeed a rare termite mimic. It is our hope that others will continue the search until the species is rediscovered and can be studied more definitively.

\footnotetext{
1 Theodor Boveri Institut für Biowissenschaften (Biozentrum) der Universität, Lehrstuhl für Zoologie II, Am Hubland, 8700 Würzburg, Germany

2 Museum of Comparative Zoology, Harvard University, 26 Oxford Street, Cambridge, Massachusetts 02138-2902, USA

Manuscript received 1 March 1992
} 
Pheidole nasutoides, new species

(Figures 1,2)

Diagnosis. A small species closest to $P$. defecta Santschi of Guatemala, and in lesser degree to members of the P. tepicana group of the southwestern United States and Mexican Plateau. $P$. nasutoides shares with these species a relatively small size in both castes; a single pair of hypostomal teeth in the major worker; and many aspects of general habitus, including the mesonotal hump reduced or absent in profile in both castes. It is distinct from members of the tepicana group in being completely dimorphic instead of trimorphic (i.e., with one rather than two major castes) and in many details of sculpturing. The major worker differs from that of defecta in the possession of a small but distinct mesonotal hump, proportionately longer petiolar peduncle, a larger propodeal spiracle, and extensive foveolation on the mesothorax and propodeum, the surfaces of which are thereby opaque. $P$. nasutoides differs from both the tepicana group and defecta in possessing angulate humeri in the major and minor, and from all other known Pheidole in the unique coloration of the major.

Holotype major. Head Width $0.80 \mathrm{~mm}$, Head Length $0.78 \mathrm{~mm}$, Scape Length $0.50 \mathrm{~mm}$, Eye Length $0.12 \mathrm{~mm}$, Pronotal Width 0.34 $\mathrm{mm}$. Body form and pattern of surface sculpture as shown in Figure 1. Color medium yellow, except for the light brown "mask" as depicted in Figure 1 (the intensity and shape of the mask varies considerably among the major workers in the type series).

Paratype minor. (Typical specimen from holotype nest series.) Head Width $0.48 \mathrm{~mm}$, Head Length $0.54 \mathrm{~mm}$, Scape Length 0.54 $\mathrm{mm}$, Eye Length $0.10 \mathrm{~mm}$, Pronotal Width $0.32 \mathrm{~mm}$. Body form and pattern of surface sculpture as shown in Figure 2. Concolorous medium yellow. COSTA RICA: La Selva Field Station and Biological Reserve, near Puerto Viejo de Sarapiqui, Heredia Province, $10^{\circ} 26^{\prime} \mathrm{N}, 84^{\circ} 00^{\prime} \mathrm{W}, 25$ March 1985.

\section{REDESCRIPTION OF PHEIDOLE DEFECTA}

Pheidole defecta Santschi 1923 is a "lost" species, poorly described at the beginning and still known only from the unique major holotype. We have undertaken to redescribe it here, as follows. Head Width $1.10 \mathrm{~mm}$, Head Length $1.10 \mathrm{~mm}$, Scape Length 

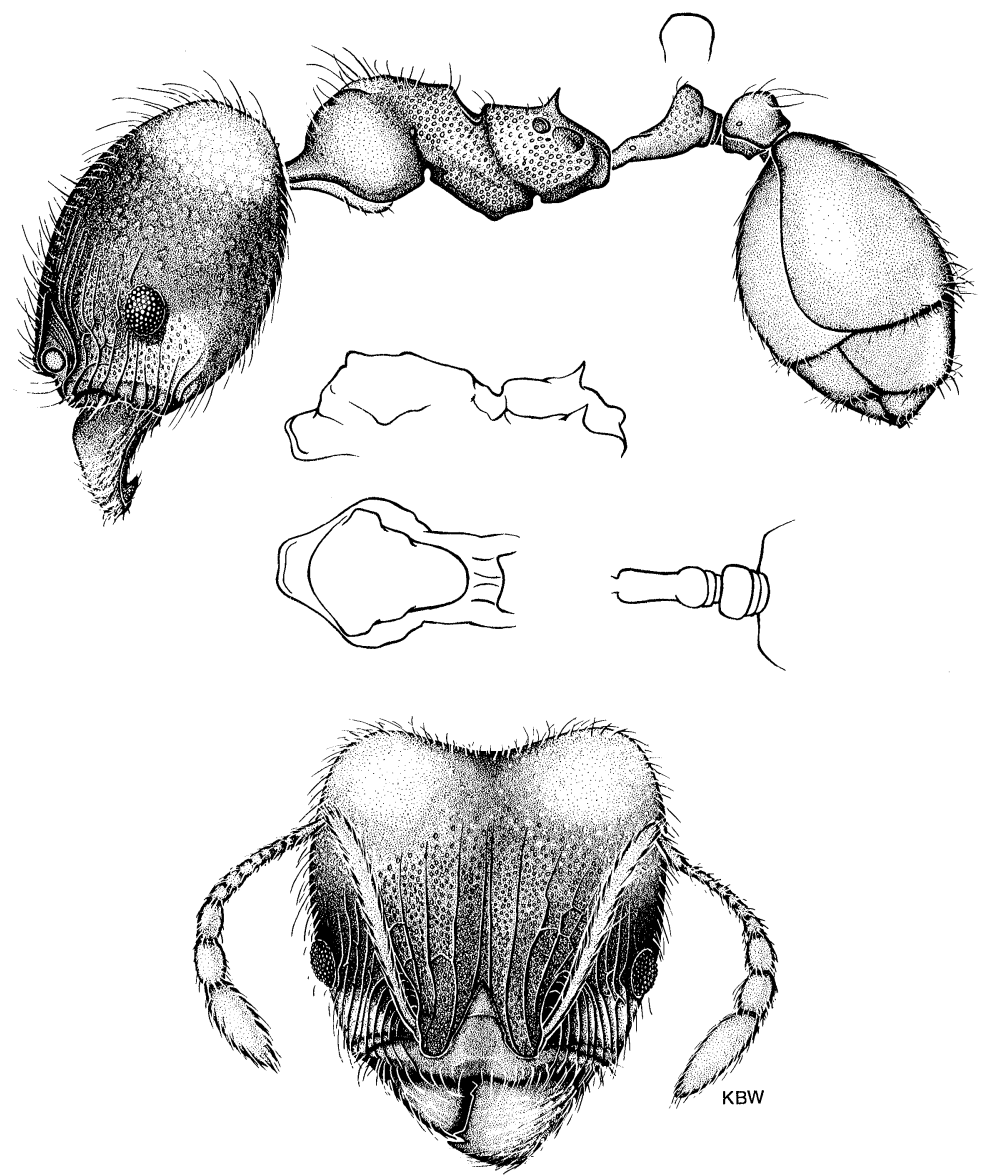

Fig. 1. Holotype major worker of Pheidole nasutoides, new species. The deeper stippling on the head represents the extent of the light brown "mask" of the face.

$0.64 \mathrm{~mm}$, Eye Length $0.16 \mathrm{~mm}$, Pronotal Width $0.56 \mathrm{~mm}$. Body form as depicted in Figure 3. Especially notable are the short, thick petiolar peduncle and the relatively sparse pilosity. Small patches occur on the mesothorax and sides of the petiole, as well as in the intercarinular spaces between the eye and frontal lobes, that are shallowly foveolate and feebly shining to subopaque. The 

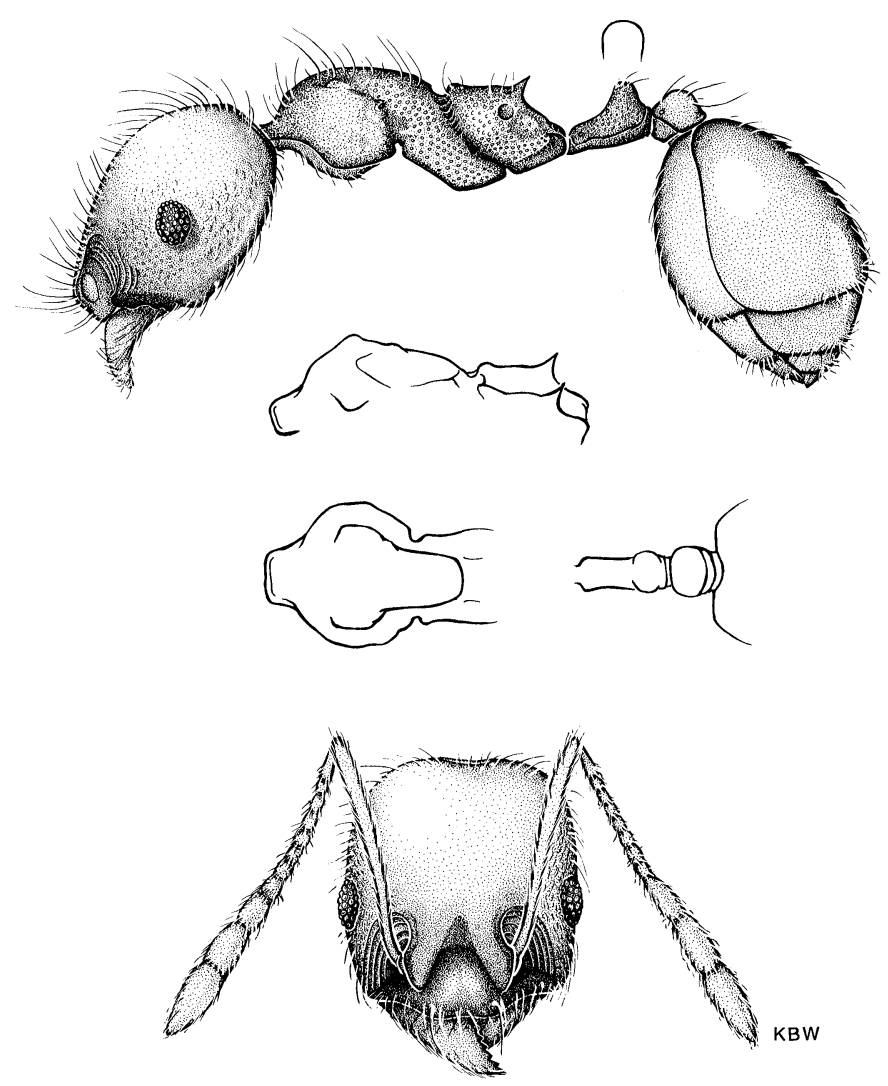

Fig. 2. Paratype minor worker of Pheidole nasutoides, new species.

remainder of the body is smooth and shining. Concolorous medium yellow. Type locality: "Guatemala," no further data.

$P$. defecta is possibly a member of the tepicana group, although the lack of other specimens prevents us from judging whether the worker caste is polymorphic. Also, if defecta does belong in this group, it is the southernmost known member. If it is fully 

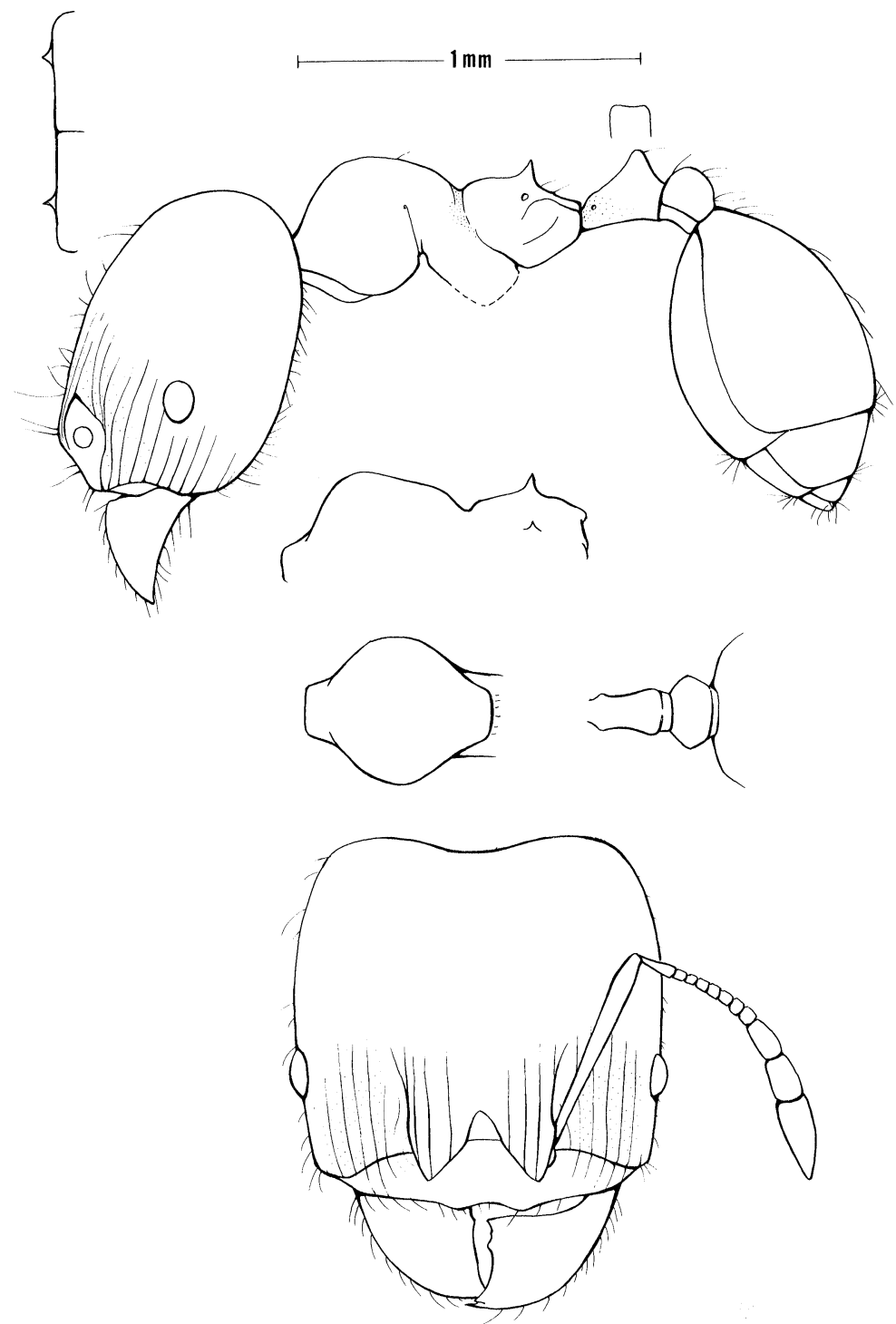

Fig. 3. Unique holotype major worker of Pheidole defecta Santschi. The stippling demarcates limited areas of shallow foveolation. The bidentate hypostomal border, similar in appearance to that of $P$. nasutoides, is depicted in the upper lefthand corner. 
dimorphic, the usual condition of Pheidole and one displayed by $P$. nasutoides, then it may be regarded as phyletically close to nasutoides and forming with that species a small, distinctive Central American species group (the "defecta group").

\section{NATURAL History}

The type colony was found nesting in a round mass of dried, thatch-like vegetation about 1.5 meters up in the moderately dense foliage of a small tree, which was located at the edge of a secondgrowth forest bordering the open experimental fields of the La Selva station. When the nest was disturbed, more than a hundred major and minor workers of $P$. nasutoides rushed out and ran in erratic looping patterns to form a spreading wave away from the nest (as shown in Fig. 4). The resemblance of the majors to Nasutitermes nasute soldiers under similar circumstances was remarkable. In particular, the mask of the Pheidole majors is roughly shaped like that of the nasute termites and contrasts with the light remainder of the body in the same way (Fig. 5). The illusion was heightened when the ants were in motion, creating a Nasutitermeslike gestalt. Hölldobler, who discovered the nest, in fact first thought that the ants were Nasutitermes and nearly passed them by. During the brief time the colony was observed live in the laboratory, the resemblance remained close. Otherwise, the colony seemed typical for a species of Pheidole. Adult males were present, but neither alate nor dealate queens were recovered.

We remain puzzled by our failure to locate other $P$. nasutoides nests despite prolonged effort in the La Selva area. It is possible that the species is simply very rare, existing in extremely sparse populations. Alternatively, it may be normally a dweller of the high canopy, a zone we did not explore. The nest found was at the edge of a disturbed forest patch, and might have fallen from a higher location. 


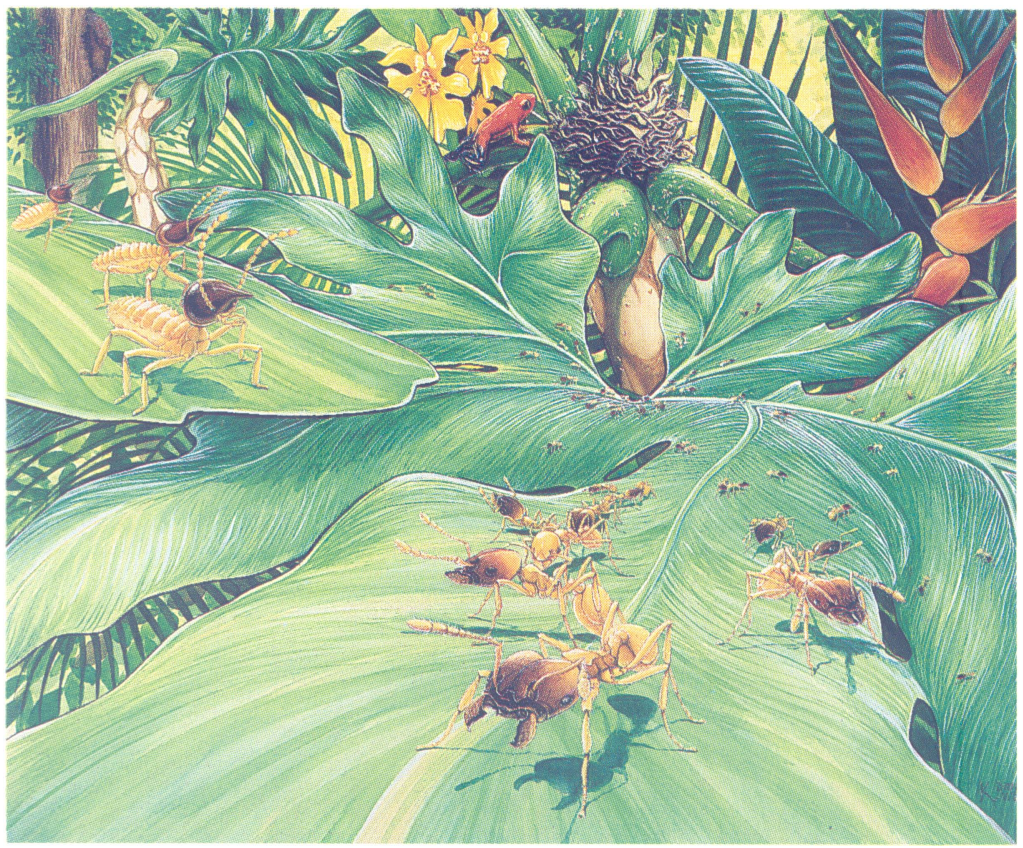

Fig. 4. Reconstruction, with imaginary details in the surrounding vegetation and animals, of the type colony of Pheidole nasutoides at La Selva, Costa Rica. The nest of the ants has been disturbed (in this hypothetical scenario) by a Dendrobates frog, a species known to feed on ants. Both majors and minors swarm out and run swiftly in erratic looping paths over the vegetation. This motion, together with their unique color pattern, causes the large-headed major workers to resemble nasute soldiers of the termite genus Nasutitermes. Several of the termite soldiers, on a foraging expedition, pause on a leaf to the left.

\section{ACKNOWLEDGEMENTS}

The illustrations were prepared by Katherine Brown-Wing. Research was supported in part by a grant from the U.S. National Science Foundation No. DEB-89-15314. 

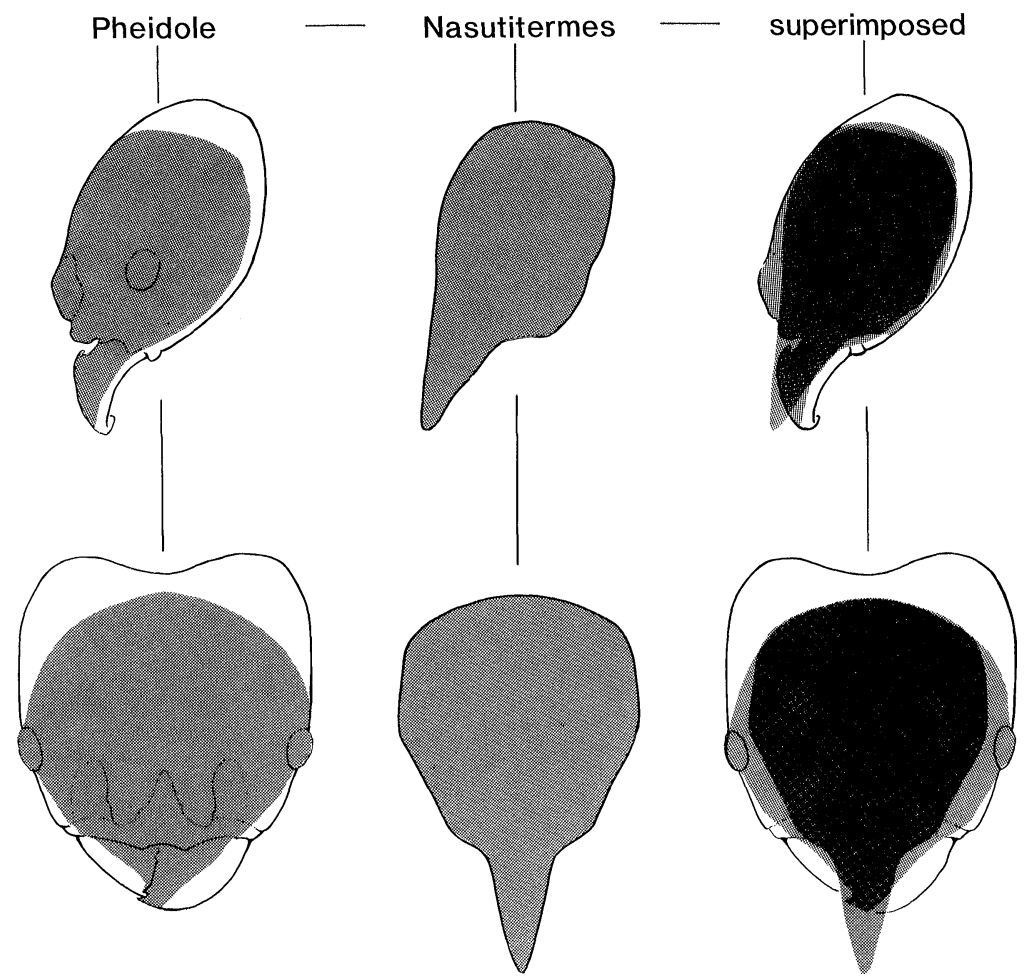

Fig. 5. The dark areas of the head capsules of a P. nasutoides major and a Nasutitermes nasute soldier, depicted separately and superimposed.

\section{REFERENCES}

HÖLLDOBLER, B. AND E. O. WILSON

1990. The Ants. Belknap Press of Harvard University Press, Cambridge, Mass. xi $+732 \mathrm{pp}$.

SANTSCHI, F.

1923. Pheidole et quelques autres fourmis néotropiques. Ann. Ent. Soc. Belg., 63: 45-69 (description of $P$. defecta: pp. 54-55). 

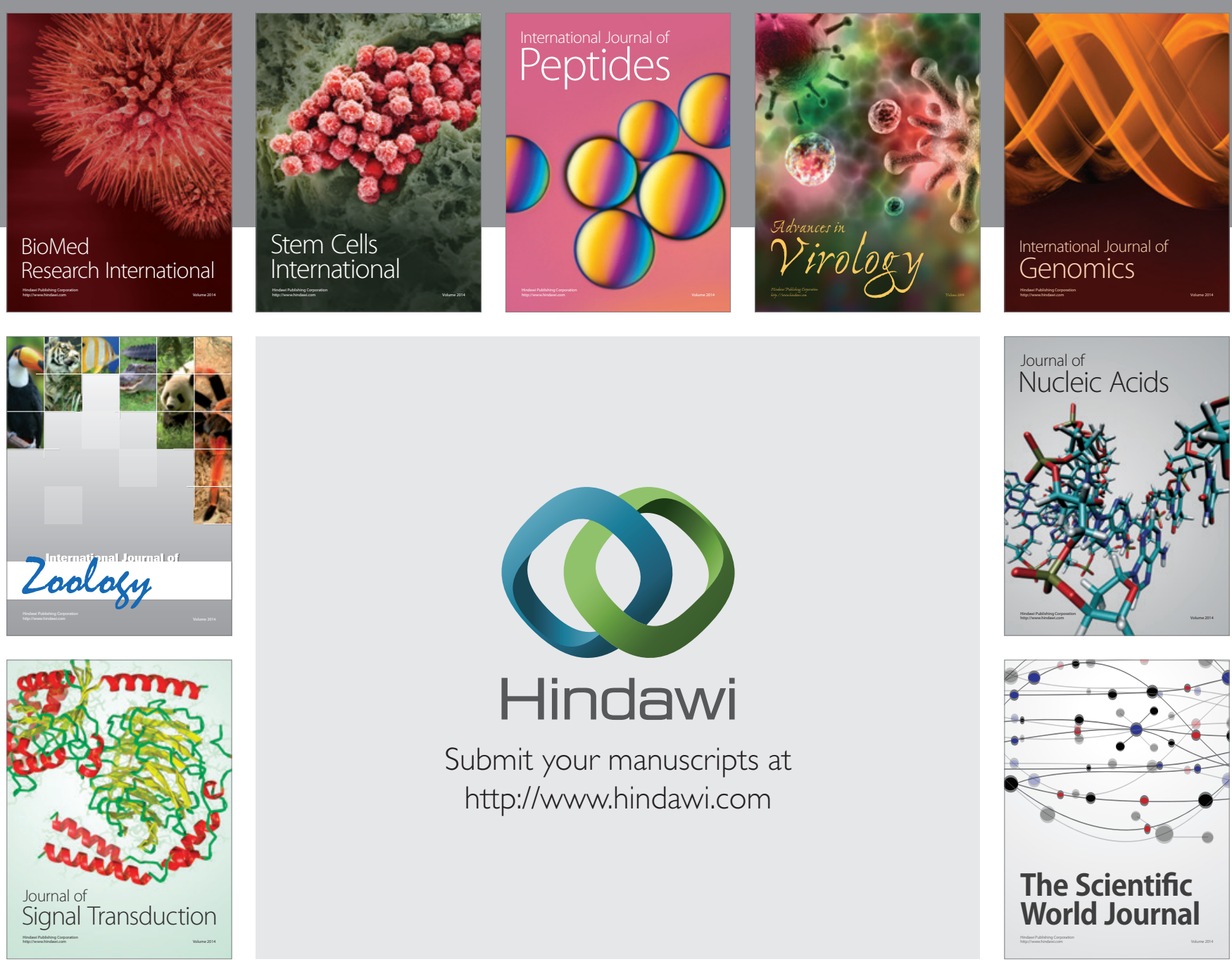

Submit your manuscripts at

http://www.hindawi.com
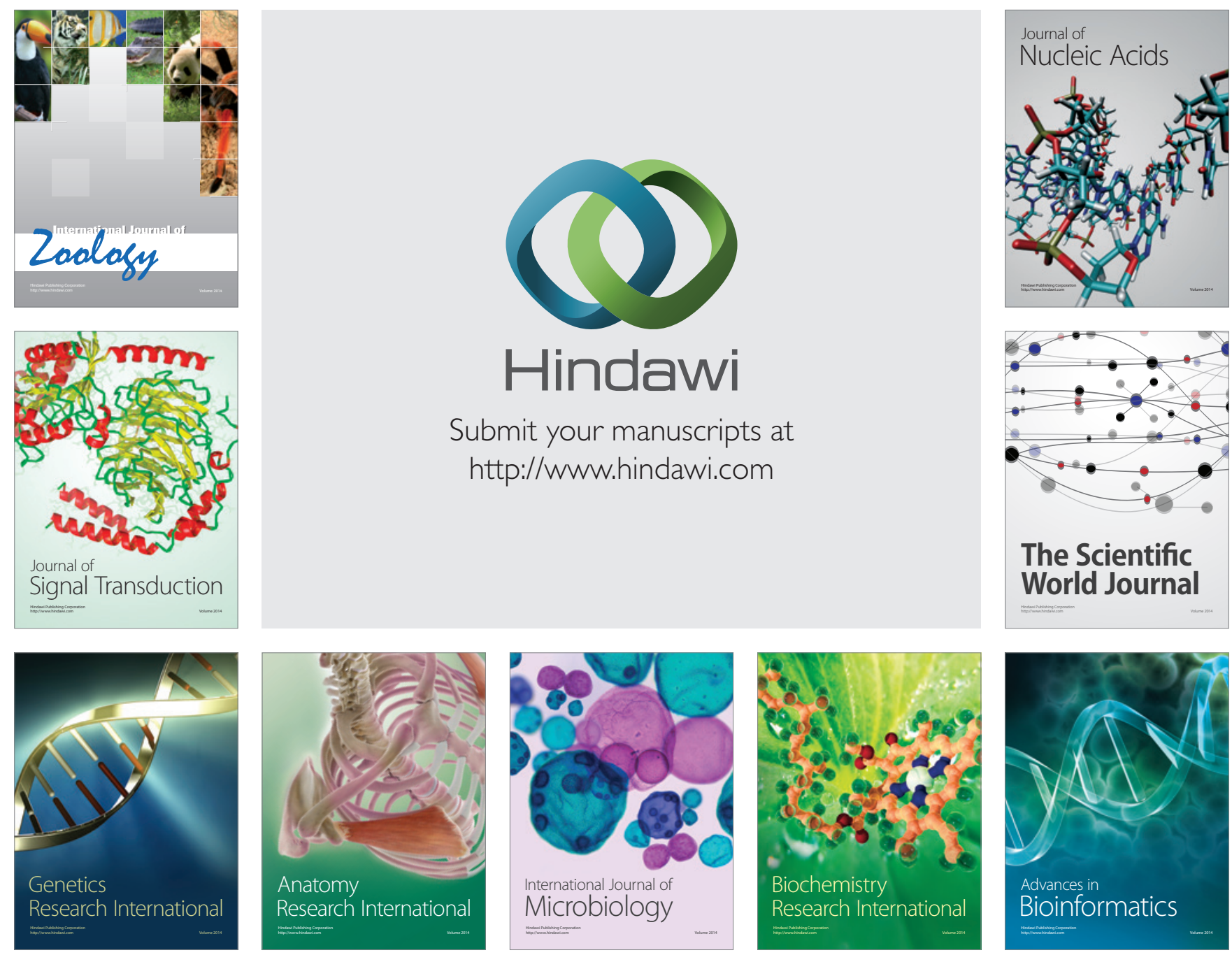

The Scientific World Journal
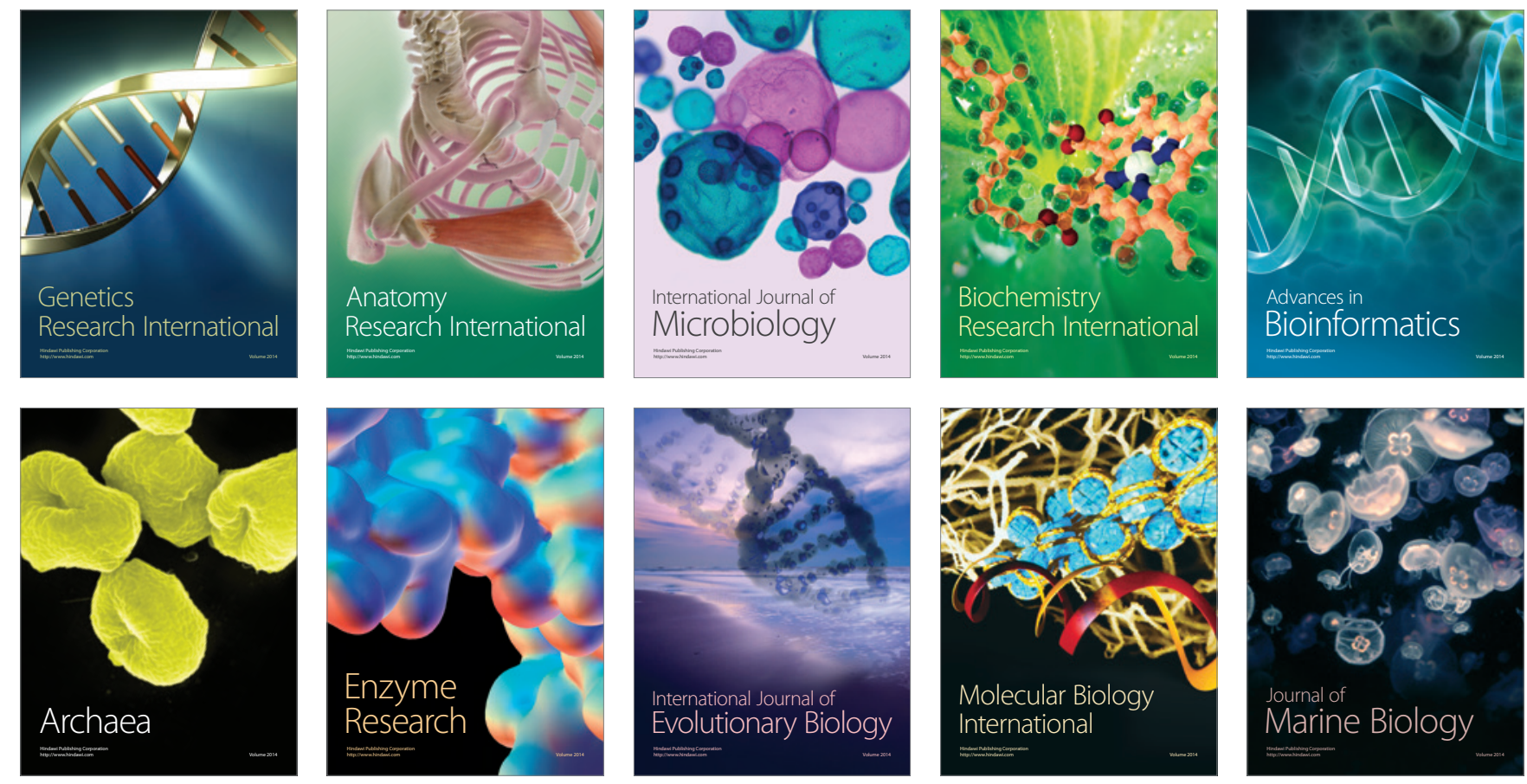